\title{
ON A CLASS OF CERTAIN ANALYTIC FUNCTIONS OF COMPLEX ORDER
}

\author{
VINOD KUMAR, S. L. SHUKLA AND A. M. CHAUDHARY
}

\begin{abstract}
We introduce a class, namely, $F_{n}(b, M)$ of certain analytic functions. For this class we determine coefficient estimate, sufficient condition in terms of coefficients, maximization theorme concerning the coefficients, radius problem and a. necessary and sufficient condition in terms of convolution. Our results generalize and correct some results of Nasr and Aouf ([2],[3]).
\end{abstract}

\section{Introduction.}

Let $\mathrm{A}$ denote the class of functions $f(z)=z+\sum_{k=2}^{\infty} a_{k} z^{k}$ which are analytic in the unit $\operatorname{disc} U=\{z:|z|<1\}$. We use $B$ to denote the class of analytic functions $\omega(z)$ in $U$ satisfying the conditions $\omega(0)=0$ and $|\omega(z)|<1$ for $z \in U$. Let $f(z)=z+\sum_{k=2}^{\infty} a_{k} z^{k}$ and $g(z)=z+\sum_{k=2}^{\infty} b_{k} z^{k}$ belong to $A$, then the convolution or Hadamard product of $f(z)$ and $g(z)$, denoted by $f(z) * g(z)$, is defined by

$$
f(z) * g(z)=z+\sum_{k=2}^{\infty} a_{k} b_{k} z^{k}, z \in U .
$$

Let $n \in N_{0}=\{0,1,2,3, \ldots\}$. The $n^{\text {th }}$ order Ruscheweyh derivative (see, [1]) of $f(z)$, denoted by $D^{n} f(z)$, is defined by

$$
D^{n} f(z)=\frac{z\left(z^{n-1} f(z)\right)^{(n)}}{n !}, n \in N_{0}
$$

Ruscheweyh [9] determined that

$$
D^{n} f(z)=\frac{z}{(1-z)^{n+1}} * f(z)
$$

We now introduce a class, namely, $F_{n}(b, M)$ of analytic functions.

Received July 27, 1987.

1980 Mathematics subject classification $30 \mathrm{C} 45$.

This work is supported by the University Grants Commission under grant number F.8-6/85 (SR III). 

and

A function $f(z)$ of $A$ belongs to the class $F_{n}(b, M)$ if and only if $\frac{D^{n} f(z)}{z} \neq 0$ in $U$

$$
\left|\frac{(b-1)+z \frac{\left(D^{n} f(z)\right)^{\prime}}{D^{n} f(z)}}{b}-M\right|<M, z \in U
$$

where $M>\frac{1}{2}$ and $b$ is any non-zero complex number.

It follows by [6] that $g(z) \in F_{0, M}$ if and only if for $z \in U$

$$
z \frac{g^{\prime}(z)}{g(z)}=\frac{1+\omega(z)}{1-m \omega(z)}
$$

where $m=1-\frac{1}{M}$ and $\omega(z) \in B$.

One can easily show that $f(z) \in F_{n}(b, M)$ if and only if there is a function $g(z) \in$ $F_{0, M}$ such that

$$
D^{n} f(z)=z\left[\frac{g(z)}{z}\right]^{b}
$$

Thus from (1.5) and (1.6) it follows that $f(z) \in F_{n}(b, M)$ if and only if for $z \in U$

$$
z \frac{\left(D^{n} f(z)\right)^{\prime}}{D^{n} f(z)}=\frac{1+\{b(1+m)-m\} \omega(z)}{1-m \omega(z)}
$$

where $\omega(z) \in B$ and $m=1-\frac{1}{M}\left(M>\frac{1}{2}\right)$.

By giving specific values to $n, b$ and $M$, we obtain the following important subclasses studied by various researchers in earlier works:

(i) Taking $n=0$, the class $F_{n}(b, M)$ coincides with the class $F(b, M)$ studied by Nasr and Aouf [2].

(ii) Taking $n=1$, the class $F_{n}(b, M)$ coincides with the class $G(b, M)$ investigated by Nasr and Aouf [3].

(iii) Taking $n=0, b=\cos \lambda e^{-i \lambda}\left(|\lambda|<\frac{\pi}{2}\right)$ and $M=\infty$, the class $F_{n}(b, M)$ coincides with the class $S^{\lambda},|\lambda|<\frac{\pi}{2}$ introduced by Špacek [14].

(iv) Taking $n=0$ and $M=\infty$, the class $F_{n}(b, M)$ coincides with the class $S(1-b)$ investigated by $\mathrm{Nasr}$ and Aouf [4].

(v) Taking $n=0$ and $b=\cos \lambda e^{-i \lambda}$, the class $F_{n}(b, M)$ coincides with the class $F_{\lambda, M}|\lambda|<\frac{\pi}{2}$ studied by Kulshrestha [6].

(vi) Taking $n=1$ and $b=\cos \lambda e^{-i \lambda}$, the class $F_{n}(b, M)$ coincides with the class $G_{\lambda, M}|\lambda|<\frac{\pi}{2}$ investigated by Kulshrestha [7].

(vii) Taking $n=1, b=\cos \lambda e^{-i \lambda}\left(|\lambda|<\frac{\pi}{2}\right)$ and $M=\infty$, the class $F_{n}(b, M)$ coincides with the class $C^{\lambda},|\lambda|<\frac{\pi}{2}$ introduced by Robertson [11].

(viii) Taking $n=1$ and $M=\infty$, the class $F_{n}(b, M)$ coincides with the class $C(b)$ introduced by Wiatrowski [15] and studied by Nasr and Aouf [5]. 
(ix) Taking $b=1$ and $M=\infty$, the class $F_{n}(b, M)$ coincides with the class $R_{n}$ introduced and studied by Singh and Singh [12].

(x) Taking $b=1-\beta(0 \leq \beta<1), n=1-2 \alpha(0 \leq \alpha<1)$ and $M=\infty$, the class $F_{n}(b, M)$ coincides with the class $R(\alpha, \beta)$ introduced and studied by -Sheil-Small, Silverman and Silvia [13].

(xi) Taking $b=1-\alpha, n=1-2 \alpha(0 \leq \alpha<1)$ and $M=\infty$, the class $F_{n}(b, M)$ coincides with the class $R_{\alpha}$ introduced and studied by Ruscheweyh [10].

From the definitions of the classes $F(b, M)$ and $F_{n}(b, M)$, we observe that

$$
f(z) \in F_{n}(b, M) \Longleftrightarrow D^{n} f(z) \in F(b, M) \text {. }
$$

The purpose of the present paper is to determine coefficient estimate, sufficient condition in terms of coefficients for a function to belong to $F_{n}(b, M)$ and maximization of $\left|a_{3}-\mu a_{2}^{2}\right|$ over the class $F_{n}(b, M)$ for complex value of $\mu$. Further we obtain the radius of disc in which $\operatorname{Re}\left\{z \frac{\left(D^{n} f(z)\right)^{\prime}}{D^{n} f(z)}\right\}>0$, whenever $f(z)$ belongs to $F_{n}(b, M)$ and a necessary and sufficient condition in terms of convolution for a function to be in $F_{n}(b, M)$. We also obtain the correct form of Maximization theorems concerning the coefficients for the classes $F(b, M)$ and $G(b, M)$, by taking $n=0$ and $n=1$, in our maximization theorem for the class $F_{n}(b, M)$ respectively.

Our results generalize the some results of Nasr and Aouf ([2], [3], [4], [5]), Kulshrestha ([6], [7]), Ruscheweyh [10], Robertson [11], Singh and Singh [12], Sheil-Small, Silverman and Silvia [13], Špace ̌ [14] and Wiatrowski [15].

\section{Preliminary lemma}

In our investigation we require the following Lemma due to Keogh and Merkes [8]:

Lemma 2.1. Let $\omega(z)=\sum_{k=1}^{\infty} c_{k} z^{k}$ belongs to $B$. If $\mu$ is any complex number then

$$
\left|c_{2}-\mu c_{1}^{2}\right| \leq \max \{1,|\mu|\} \text {. }
$$

Equality may be attained with the functions $\omega(z)=z^{2}$ and $\omega(z)=z$.

\section{Coefficient estimate}

Theorem 3.1. If $f(z)=z+\sum_{k=2}^{\infty} a_{k} z^{k}$ belongs to $F(b, M)$ and

$$
L=|b|^{2}(1+m)+2 m(j-1) \operatorname{Re}(b)+(m-1)(j-1)^{2},
$$

then

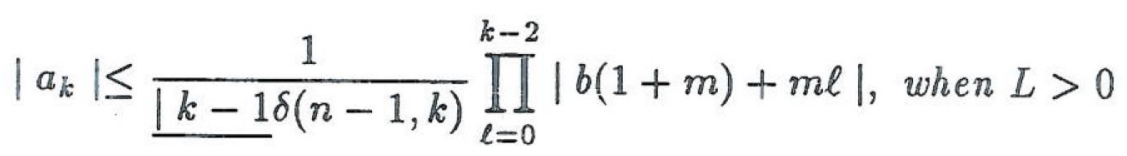

and

$$
\left|a_{k}\right| \leq \frac{1}{(k-1) \delta(n-1, k)}|b(1+m)| \text {, when } L \leq 0 \text {, }
$$

where $m=1-\frac{1}{M}\left(M>\frac{1}{2}\right)$ and $\delta(n, k)=\left(\begin{array}{c}n+k \\ n+1\end{array}\right)$. 
The inequalities (3.1) and (3.2) are sharp.

Proof. Since $f(z) \in F_{n}(b, M)$, so from (1.7) we have that

$$
z \frac{\left(D^{n} f(z)\right)^{\prime}}{D^{n} f(z)}=\frac{1+\{b(1+m)-m\} \omega(z)}{1-m \omega(z)}
$$

or

$$
\sum_{j=2}^{\infty}(j-1) \delta(n-1, j) a_{j} z^{j}=\left[b(1+m) z+\sum_{j=2}^{\infty}\{b(1+m)+m(j-1)\} \delta(n-1, j) a_{j} z^{j}\right] \omega(z)
$$

or

$$
\begin{aligned}
\sum_{j=2}^{k}(j-1) \delta(n-1, j) a_{j} z^{j}+\sum_{j=k+1}^{\infty} d_{j} z^{j}= & {\left[b(1+m) z+\sum_{j=2}^{k-1}\{b(1+m)\right.} \\
& \left.+m(j-1)\} \delta(n-1, j) a_{j} z^{j}\right] \omega(z),
\end{aligned}
$$

where $d_{j}$ 's are some complex numbers. Then since $|\omega(z)|<1$, we have

$$
\begin{aligned}
\mid b(1+m) z & +\sum_{j=2}^{k-1}\{b(1+m)+m(j-1)\} \delta(n-1, j) a_{j} z^{j} \mid \\
& \geq\left|\sum_{j=2}^{k}(j-1) \delta(n-1, j) a_{j} z^{j}+\sum_{j=k+1}^{\infty} d_{j} z^{j}\right| .
\end{aligned}
$$

Squaring both sides of (3.3) and integrating round $|z|=r<1$, we get, after taking the limit when $r \rightarrow 1$

$$
\begin{gathered}
(k-1)^{2}\{\delta(n-1, k)\}^{2}\left|a_{k}\right|^{2} \leq|b(1+m)|^{2}+\sum_{j=2}^{k-1}\left[|b(1+m)+m(j-1)|^{2}\right. \\
\left.-(j-1)^{2}\right]\{\delta(n-1, j)\}^{2}\left|a_{j}\right|^{2}
\end{gathered}
$$

On solving, we get

$$
\begin{aligned}
& (k-1)^{2}\{\delta(n-1, k)\}^{2}\left|a_{k}\right|^{2} \\
& \leq|b(1+m)|^{2}+\sum_{j=2}^{k-1}\left[( 1 + m ) \left\{|b|^{2}(1+m)\right.\right. \\
& \left.\left.\quad+2 m(j-1) \operatorname{Re}(b)+(m-1)(j-1)^{2}\right\}\right]\{\delta(n-1, j)\}^{2}\left|a_{j}\right|^{2}
\end{aligned}
$$

or

$$
\begin{aligned}
& (k-1)^{2}\{\delta(n-1, k)\}^{2}\left|a_{k}\right|^{2} \leq|b(1+m)|^{2} \\
& \quad+\sum_{j=2}^{k-1}(1+m) L\{\delta(n-1, j)\}^{2}\left|a_{j}\right|^{2},
\end{aligned}
$$


where

$$
L=\left\{|b|^{2}(1+m)+2 m(j-1) \operatorname{Re}(b)+(m-1)(j-1)^{2}\right\} .
$$

Now we consider the following two cases:

Cases I: When $L>0$. In this case (3.4) gives successively for $k=2,3,4, \ldots$

$$
\left|a_{2}\right| \leq \frac{|b(1+m)|}{\delta(n-1,2)},\left|a_{3}\right| \leq \frac{|b(1+m)||b(1+m)+m|}{2 \delta(n-1,3)}
$$

and hence by induction

$$
\left|a_{k}\right| \leq \frac{1}{\left|\underline{k-1 \delta(n-1, k)} \prod_{l=0}^{k-2}\right| b(1+m)+m l \mid . ~}
$$

The above inequality is sharp for the function $f(z)$ defined by

$$
D^{n} f(z)= \begin{cases}\frac{z}{(1-m z)^{b(1+m) / m}}, & \text { for } m \neq 0 \\ z \exp (b z), & \text { for } m=0 .\end{cases}
$$

Case II: When $L \leq 0$. In this case (3.4) gives

$$
\left|a_{k}\right| \leq \frac{1}{(k-1) \delta(n-1, k)}|b(1+m)| .
$$

This inequality is sharp.

Theorem 3.2. Let $f(z)=z+\sum_{k=2}^{\infty} a_{k} z^{k}$. If for some $n \geq 0$ and $A, B$ with $-1 \leq$ $B<A \leq 1$

$$
\sum_{k=2}^{\infty}[(k-1)+|b(1+m)+m(k-1)|] \delta(n-1, k)\left|a_{k}\right| \leq|b(1+m)|
$$

holds, then $f(z)$ belongs to $F_{n}(b, m)$, where $\delta(n, k)=\left(\begin{array}{l}n+k \\ n+1\end{array}\right)$ and $m=1-\frac{1}{M}\left(M>\frac{1}{2}\right)$.

Proof. Suppose that the inequality (3.5) holds. Since $f(z)=z+\sum_{k=2}^{\infty} \delta(n-$ $1, k) a_{k} z^{k}$, we have for $z \in U$

$$
\begin{aligned}
& \left|z\left(D^{n} f(z)\right)^{\prime}-D^{n} f(z)\right|-\left|b(1+m) D^{n} f(z)+m\left\{z\left(D^{n} f(z)\right)^{\prime}-D^{n} f(z)\right\}\right| \\
= & \left|\sum_{k=2}^{\infty}(k-1) \delta(n-1, k) a_{k} z^{k}\right|-\mid b(1+m)\left\{z+\sum_{k=2}^{\infty} \delta(n-1, k) a_{k} z^{k}\right\} \\
& \quad+m \sum_{k=2}^{\infty}(k-1) \delta(n-1, k) a_{k} z^{k} \mid \\
\leq & \sum_{k=2}^{\infty}(k-1) \delta(n-1, k)\left|a_{k}\right| r^{k}
\end{aligned}
$$




$$
\begin{aligned}
& \quad-\left\{|b(1+m)| r-\sum_{k=2}^{\infty}|b(1+m)+m(k-1)| \delta(n-1, k)\left|a_{k}\right| r^{k}\right\} \\
& =\sum_{k=2}^{\infty}[(k-1)+|b(1+m)+m(k-1)|] \delta(n-1, k)\left|a_{k}\right| r^{k}-|b(1+m)| r \\
& \leq \sum_{k=2}^{\infty}[(k-1)+|b(1+m)+m(k-1)|] \delta(n-1, k)\left|a_{k}\right|-|b(1+m)| \\
& \leq 0, \text { by (3.5) }
\end{aligned}
$$

Hence it follows that

$$
\left|\frac{\left\{z \frac{\left(D^{n} f(z)\right)^{\prime}}{D^{n} f(z)}-1\right\}}{b(1+m)+m\left\{z \frac{\left(D^{n} f(z)\right)^{\prime}}{D^{n} f(z)}-1\right\}}\right|<1, z \in U .
$$

Letting

$$
\omega(z)=\frac{\left\{z \frac{\left(D^{n} f(z)\right)^{\prime}}{D^{n} f(z)}-1\right\}}{b(1+m)+m\left\{\frac{z\left(D^{n} f(z)\right)^{\prime}}{D^{n} f(z)}-1\right\}},
$$

then $\omega(0)=0, \omega(z)$ is analytic in $|z|<1$ and $|\omega(z)|<1$. Hence we have

$$
\frac{z\left(D^{n} f(z)\right)^{\prime}}{D^{n} f(z)}=\frac{1+\{b(1+m)-m\} \omega(z)}{1-m \omega(z)}
$$

which shows that $f(z)$ belongs to $F_{n}(b, M)$.

4. Maximization of $\left|a_{3}-\mu a_{2}^{2}\right|$

Theorem 4.1. If $f(z)=z+\sum_{k=2}^{\infty} a_{k} z^{k}$ belongs to $F_{n}(b, M)$ and $\mu$ is any complex number then

$$
\left|a_{3}-\mu a_{2}^{2}\right| \leq \frac{|b(1+m)|}{2 \delta(n-1,3)} \max \{1,|d|\}
$$

where

$$
d=\frac{2 \mu b(1+m) \delta(n-1,3)-\{b(1+m)+m\}\{\delta(n-1,2)\}^{2}}{\{\delta(n-1,2)\}^{2}}
$$

This inequality is sharp for each $\mu$.

Proof. Since $f(z)$ belongs to $F_{n}(b, M)$, so that from $(1.7)$ we have

$$
z \frac{\left(D^{n} f(z)\right)^{\prime}}{D^{n} f(z)}=\frac{1+\{b(1+m)-m\} \omega(z)}{1-m \omega(z)}
$$


or

$$
\begin{aligned}
\omega(z)= & \frac{\left\{z\left(D^{n} f(z)\right)^{\prime}-D^{n} f(z)\right\}}{\left[\{b(1+m)-m\} D^{n} f(z)+m z\left(D^{n} f(z)\right)^{\prime}\right]} \\
= & \frac{\sum_{k=2}^{\infty}(k-1) \delta(n-1, k) a_{k} z^{k-1}}{\left[b(1+m)+\sum_{k=2}^{\infty}\{b(1+m)+m(k-1)\} \delta(n-1, k) a_{k} z^{k-1}\right]} \\
= & \frac{\sum_{k=2}^{\infty}(k-1) \delta(n-1, k) a_{k} z^{k-1}}{b(1+m)} \\
& {\left[1+\frac{\sum_{k=2}^{\infty}\{b(1+m)+m(k-1)\} \delta(n-1, k) a_{k} z^{k-1}}{b(1+m)}\right]^{-1} }
\end{aligned}
$$

Equating the coefficients of $z$ and $z^{2}$ on both sides, we get

$$
a_{2}=\frac{b(1+m) c_{1}}{\delta(n-1,2)}
$$

and

$$
a_{3}=\frac{b(1+m)}{2 \delta(n-1,3)}\left[c_{2}+\{b(1+m)+m\} c_{1}^{2}\right]
$$

Hence

$$
a_{3}-\mu a_{2}^{2}=\frac{b(1+m)}{2 \delta(n-1,3)}\left[c_{2}-d c_{1}^{2}\right]
$$

where

$$
d=\frac{2 \mu b(1+m) \delta(n-1,3)-\{b(1+m)+m\}\{\delta(n-1,2)\}^{2}}{\{\delta(n-1,2)\}^{2}}
$$

Taking modulus both sides in (4.4), we have

$$
\left|a_{3}-\mu a_{2}^{2}\right|=\frac{|b(1+m)|}{2 \delta(n-1,3)}\left|c_{2}-d c_{1}^{2}\right| \text {. }
$$

Using Lemma 2.1 in (4.5), we have

$$
\left|a_{3}-\mu a_{2}^{2}\right| \leq \frac{|b(1+m)|}{2 \delta(n-1,3)} \max \{1,|d|\}
$$

Since the inequality (2.1) is sharp, so that the inequality (4.1) must also be sharp.

Corollary. If $f(z)$ belongs to $F_{n}(b, M)$, then

$$
\left|a_{2}\right| \leq \frac{|b|(1+m)}{\delta(n-1,2)}
$$

and

$$
\left|a_{3}\right| \leq \frac{|b|(1+m)}{2 \delta(n-1,3)} \max \{1,|b(1+m)+m|\} .
$$

The inequalities (4.6) and (4.7) follow directly from (4.2) and (4.3) respectively. 
Remarks.

(i) We obtain the maximization of $\left|a_{3}-\mu a_{2}^{2}\right|$ over the class $F(b, M)$ which is studied by Nasr and Aouf [2], in correct form, by taking $n=0$ in our maximization of $\left|a_{3}-\mu a_{2}^{2}\right|$ over the class $F_{n}(b, M)$.

(ii) We also obtain the maximization of $\left|a_{3}-\mu a_{2}^{2}\right|$ over the class $G(b, M)$ which is studied by $\mathrm{Nasr}$ and Aouf [3], in correct form, by taking $n=1$ in our maximization of $\left|a_{3}-\mu a_{2}^{2}\right|$ over the class $F_{n}(b, M)$.

\section{Radius theorem}

The following theorem may by obtained with the help of (1.8) and Lemma 3 of Nasr and Aouf [2].

Theorem 5.1. Let $f(z)=z+\sum_{k=2}^{\infty} a_{k} z^{k}$ belongs to $F_{n}(b, M)$. Then

$$
\operatorname{Re}\left\{\frac{z\left(D^{n} f(z)\right)^{\prime}}{D^{n} f(z)}\right\}>0 \text { for }|z|<r_{n}
$$

where

$$
r_{n}=2\left\{|b|(1+m)+\left[|b|^{2}(1+m)^{2}-4\left\{\operatorname{Re}(b)\left(\frac{1+m}{m}\right)-1\right\}\right]^{\frac{1}{2}}\right\}^{-1}
$$

Remarks. In the above mentioned result

(i) Putting $n=0$, we get the sharp radius of starlikeness of the class $F(b, M)$ which is studied by Nasr and Aouf [2].

(ii) Putting $n=1$, we get the sharp radius of convexity of the class $G(b, M)$ which is investigated by Nasr and Aouf [3].

\section{Necessary and sufficient condition}

Theorem 6.1. A function $f(z)$ belongs to the class $F_{n}(b, M)$ if and only if

$$
f(z) *\left[\frac{z+\left[\frac{(n+1)+x\{b(1+m)-m(n+1)\}}{-b(1+m) x}\right] z^{2}}{(1-z)^{n+2}}\right] \neq 0
$$

in $0<|z|<1$, where $x=1$ and $x \neq 1$.

Proof. Let $f(z)$ belongs to the class $F_{n}(b, M)$, then

$$
z \frac{\left(D^{n} f(z)\right)^{\prime}}{D^{n} f(z)} \neq \frac{1+\{b(1+m)-m\} x}{1-m x},
$$

$|x|=1$ and $x \neq 1$ in $0<|z|<1$. Equivalently

$$
(1-m x) z\left(D^{n} f(z)\right)^{\prime}-[1+\{b(1+m)-m\} x] D^{n} f(z) \neq 0 \text { in } 0<|z|<1 .
$$


We know that, by the definition of $D^{n} f(z)$

$$
z\left(D^{n} f(z)\right)^{\prime}=(n+1) D^{n+1} f(z)-n D^{n} f(z) .
$$

Using (6.3) in (6.2), we have

$$
(1-m x)\left[(n+1) D^{n+1} f(z)-n D^{n} f(z)\right]-[1+\{b(1+m)-m\} x] D^{n} f(z) \neq 0 \text { in } 0<|z|<1
$$

or

$$
f(z) *\left[\frac{-b(1+m) x z+[(n+1)+x\{b(1+m)-m(n+1)\}] z^{2}}{(1-z)^{n+2}}\right] \neq 0 .
$$

Since $x \neq 1$ and $b$ is any non-zero complex number, we have

$$
f(z) *\left[\frac{z+\left[\frac{(n+1)+x\{b(1+m)-m(n+1)\}}{-b(1+m) x}\right] z^{2}}{(1-z)^{n+2}}\right] \neq 0
$$

which is the required condition.

The converse part follows easily since all the steps can be retraced back.

\section{References}

[1] H. S. Al-Amiri, "On Ruscheweyh derivatives," Ann. Polon. Math., 38(1980), 87-94.

[2] M. A. Nasr and M. K. Aouf, "Bounded starlike functions of complex order," Proc. Indian Acad. Sci. (Math. Sci.) 92(1983).

[3] M. A. Nasr and M. K. Aouf, "Bounded convex functions of complex order," Bull. Fac. Sci. 10(1983).

[4] M. A. Nasr and M. K. Aouf, "Starlike functions of complex order," J. Naturai Sci. Math. 25(1985).

[5] M. A. Nasr and M. K. Aouf, "On convex functions of complex order," mansoura Sci. Bull. (1982), 565-582.

[6] P. K. Kulshrestha, "Distortion of spiral-like mappings," Proc. Royal Irish Acad., 73A(1973), 1-5.

[7] P. K. Kulshrestha, "Bounded Robertson function," Rend. Math., (6)9(1976), 137-150.

[8] F. R. Keogh and E. P. Merkes, "A coefficient inequality for certain classes of analytic functions," Proc. Amer. Math. Soc. 20(1969).

[9] S. Ruscheweyh, "New criteria for univalent functions," Proc. Amer. Math. Soc. 49(1975), 109-115.

[10] S. Ruscheweyh, "Linear operators between classes of prestarlike functions, comment," Math. Helve., 52(1977), 497-509.

[11] M. S. Robertson, "Univalent functions for which $z f^{\prime}(z)$ is spiral-like," Michigan Math. J., 16(1969), 97-101.

[12] Ram Singh and Sunder Singh, "Integrals of certain univalent functions," Proc. Amer. Math. Soc., $77(1979), 336-340$.

[13] T. Sheil-Small, H. Silverman and E. M. Silvia, "Convolution multipliers and star-like functions," J. Analyse Math. 41(1982), 181-192.

[14] L. Špaceǩ, "Prispeekk teorii funki, Prostych," casopis Pest Math. Fys., 62(1933), 12-19.

[15] P. Wiatrowski, "The coefficients of a certain family of holomorphic functions," Zeszyty Nauk. Univ. todzk, Nauki. Math. Przyrod. ser II, zeszyt (39) Math. (1971), 75-85.

Department of Mathematics, Christ Church College, Kanpur-208001 (U.P.), India.

Department of Mathematics, Janta College, Bakewar-206124, Etawah (U.P.), India. 